\title{
New standardized nursing cooperation workflow to reduce stroke thrombolysis delays in patients with acute ischemic stroke
}

This article was published in the following Dove Press journal:

Neuropsychiatric Disease and Treatment

9 May 2017

Number of times this article has been viewed

\author{
Yan Zhou' \\ Zhuojun $\mathrm{Xu}^{2}$ \\ Jiali Liao' \\ Fangming Feng' \\ Lai $\mathrm{Men}^{3}$ \\ $\mathrm{Li} X \mathrm{u}^{2}$ \\ Yanan $\mathrm{He}^{2}$ \\ Gang $\mathrm{Li}^{2}$
}

'Nursing Department, Shanghai East Hospital, Tongji University School of Medicine, Shanghai, ${ }^{2}$ Department of Neurology, Shanghai East Hospital, Tongji University School of Medicine, Shanghai, People's Republic of China; '3Paddington

Dental Practice, London, UK
Correspondence: Gang Li

Department of Neurology, South

Campus of Shanghai East Hospital,

No 1800 Yuntai Road, Pudong New

District, Shanghai 200123, People's

Republic of China

Tel +86 I36 2169 I786

Email Ig130I@easthospital.cn
Objective: We assessed the effectiveness of a new standardized nursing cooperation workflow in patients with acute ischemic stroke (AIS) to reduce stroke thrombolysis delays.

Patients and methods: AIS patients receiving conventional thrombolysis treatment from March to September 2015 were included in the control group, referred to as T0. The intervention group, referred to as T1 group, consisted of AIS patients receiving a new standardized nursing cooperation workflow for intravenous thrombolysis (IVT) at the emergency department of Shanghai East Hospital (Shanghai, People's Republic of China) from October 2015 to March 2016. Information was collected on the following therapeutic techniques used: application or not of thrombolysis, computed tomography (CT) time, and door-to-needle (DTN) time. A nursing coordinator who helped patients fulfill the medical examinations and diagnosis was appointed to $\mathrm{T} 1$ group. In addition, a nurse was sent immediately from the stroke unit to the emergency department to aid the thrombolysis treatment.

Results: The average value of the door-to-CT initiation time was $38.67 \pm 5.21 \mathrm{~min}$ in the T0 group, whereas it was $14.39 \pm 4.35 \mathrm{~min}$ in the T1 group; the average values of CT completionto-needle time were $55.06 \pm 4.82$ and $30.26 \pm 3.66 \mathrm{~min}$; the average values of DTN time were $100.43 \pm 6.05$ and $55.68 \pm 3.62 \mathrm{~min}$, respectively; thrombolysis time was improved from $12.8 \%$ $(88 / 689)$ in the T0 group to $32.5 \%(231 / 712)$ in the T1 group (all $P<0.01)$. In addition, the new standardized nursing cooperation workflow decreased the National Institutes of Health Stroke Scale (NIHSS) scores at $24 \mathrm{~h}(P<0.01)$ (T0: prethrombolysis, 6.97 $\pm 3.98 ; 24 \mathrm{~h}$ postthrombolysis, $3.33 \pm 2.09$; 2 weeks postthrombolysis, $2.25 \pm 1.01$ and T1: prethrombolysis, $7.00 \pm 3.89 ; 24 \mathrm{~h}$ postthrombolysis, $2.60 \pm 1.66 ; 2$ weeks postthrombolysis, $2.21 \pm 1.02$ ).

Conclusion: The new standardized nursing cooperation workflow reduced stroke thrombolysis delays in patients with AIS.

Keywords: standardization, nursing, intravenous thrombolysis, door-to-needle time, stroke thrombolysis delay

\section{Introduction}

In China, stroke has been the leading cause of death among all diseases. ${ }^{1}$ According to a report by the Chinese National Stroke Registry (CNSR; 2011), ${ }^{2}$ strokes occurred in $>7$ million Chinese suffered in 2011 , and $\sim 65 \%$ of them were characterized by ischemia. Rising trends have been observed in the morbidity and mortality of stroke, which causes a heavy burden to the national health care system. ${ }^{3-5}$

Approved by the State Food and Drug Administration of the American Heart Association in 2013, intravenous thrombolysis (IVT) with recombinant tissue plasminogen activators (rtPA) is one of the effective treatments for acute ischemic 
stroke (AIS). ${ }^{6}$ Importantly, administration of thrombolysis is recommended within $1 \mathrm{~h}$, ie, door-to-needle (DTN) time $\leq 60$ min..$^{5,7,8}$ Therefore, inhospital delays diminish the therapeutic effectiveness of IVT in AIS patients. ${ }^{9}$ Indeed, in China, $<22 \%$ of all AIS patients are sent to hospital within $3 \mathrm{~h}$, of whom only $1.6 \%$ could be treated by IVT after screening tests. ${ }^{10}$ The delay in DTN time in hospitals was mainly caused by decision-making by the clinicians and laboratory tests. ${ }^{2}$

A nursing workflow consists of many organizational steps, which are indispensable for achieving excellent evidence-based patient care provided by nurses to patients with stroke. ${ }^{11}$ Unfortunately, this workflow has always been neglected in Chinese stroke patients. ${ }^{12,13}$ The implementation of a multilevel strategy, including nursing staff and measures for reducing stroke thrombolysis delays in the emergency department, is required for the hospitals throughout China. In this study, we compared the effectiveness of a newly established and standardized nursing cooperation workflow in stroke thrombolysis with that of the conventional nursing cooperation workflow.

\section{Patients and methods}

\section{Patients}

A total of 1,401 patients participated in this study. From March to September 2015, 689 patients were included in the T0 group, but only 88 of them received thrombolysis in the recommended 1-h time window due to the time wasted in administration of the conventional of thrombolytic therapy. From October 2015 to March 2016, 712 patients were included in the $\mathrm{T} 1$ group, and 231 of them finally received thrombolysis in the desired time window. AIS patients were diagnosed according to the National Guideline of Diagnosis and Treatment of AIS, and the diagnosis was confirmed by computed tomography (CT). ${ }^{14}$

The AIS patients in the $\mathrm{T} 0$ group received a traditional nursing cooperation workflow for thrombolysis treatment in the same department from January 2014 to December 2014. The AIS patients in the T1 group received a new standardized nursing cooperation workflow for IVT with rtPA in the emergency department of Shanghai East Hospital (Shanghai, People's Republic of China) from March 2015 to March 2016.

The information collected in both groups included thrombolysis rate, reach time, CT time, door-to-CT initiation time, CT completion-to-needle time, and DTN time. The study protocol was approved by the ethical committee of Shanghai East Hospital (Shanghai, People's Republic of China). Written informed consent was signed by all patients or their family members prior to enrollment.
The key points of T1 group were a nursing coordinator appointed to help fulfill all medical examinations and diagnosis, and a nurse was sent immediately from the stroke unit to the emergency department to aid in the thrombolysis treatment.

\section{Inclusion and exclusion criteria}

AIS patients were diagnosed according to the National Guideline of Diagnosis and Treatment of AIS with confirmation by CT. ${ }^{14}$ General inclusion criteria were as follows: 1) patients aged from 18 to 80 years, 2) patients meeting the criteria of AIS and the diagnosis being confirmed by cranial $\mathrm{CT}, 3$ ) persistent symptoms of brain injury $>30 \mathrm{~min}$, and 4) informed consent of patients or families.

AIS patients treated with IVT therapy were eligible if they fulfilled the following criteria: 1) patients could receive the therapy within $4.5 \mathrm{~h}$ from symptom onset and 2) patients with National Institutes of Health Stroke Scale (NIHSS) score from 4 to 25 points.

Exclusion criteria were as follows: 1) patients misdiagnosed with AIS and 2) patients already admitted in our hospital. Medical records were checked according to the same inclusion and exclusion criteria for obtaining detailed information.

\section{Newly standardized and traditional nursing cooperation workflow} Traditional nursing cooperation workflow (the nursing workflow in a collaborative effort to provide care for patients)

When AIS patients arrived at the emergency department of our hospital, the traditional nursing cooperation workflow for patients in the T0 group was characterized by the following features: 1) the patient was triaged by an emergency assessor on the basis of symptoms, such as dizziness and hemiplegia, and was then transferred to the emergency stroke specialist in the emergency department; 2) the stroke specialist assessed the condition of the patient according to the NIHSS scale, and an emergency nurse opened the "green passage" and helped the patient during the imaging examination, electrocardiogram (ECG), blood tests, and other necessary preparation procedures; 3 ) patients were examined by CT to confirm the diagnosis and exclude the presence of hemorrhagic cerebrovascular diseases and were then transferred to the stroke unit in another building; and 4) after the patient arrived at the stroke unit, a nurse of the unit began the preparation for the treatment of IVT with rtPA. 
New standardized nursing cooperation workflow (an interdisciplinary protocol to enhance communication and expediency)

When AIS patients arrived at our emergency department, a new standardized nursing cooperation workflow was performed for the patients in T1 (Figure 1). The steps were as follows: 1) the patient was triaged by an emergency assessor on the basis of symptoms, such as dizziness and hemiplegia, and was then transferred to the emergency stroke specialist doctor in the emergency department; 2) a stroke specialist determined the score of the patient according to the NIHSS scale, and a designated nurse in the Emergency Room coordinated the following workflow: opening of the green passage to shorten the waiting time of the patient and helping the patient to undergo imaging examination, ECG, and blood tests immediately upon arrival in the emergency room; furthermore, the patient was diagnosed with CT to exclude the presence of hemorrhagic cerebrovascular diseases; 3) as soon as AIS diagnosis was confirmed, a nurse in the stoke unit was informed about the "cooperation during emergency thrombolysis"; the nurse immediately brought a first-aid thrombolysis kit into the special thrombolysis room in the emergency department (within $5 \mathrm{~min}$ ); and (4) the AIS patient was transferred to the special thrombolysis room, and the doctor and the "emergency thrombolysis cooperating nurse" together administered rtPA for the treatment of the patient with IVT (Figure 1).

\section{Selection of a professional emergency thrombolysis coordinator and a cooperating nurse and establishment of a first-aid thrombolysis kit}

The key components of the new standardized nursing cooperation workflow are the professional emergency thrombolysis coordinator and the cooperating nurse. The coordinator was an emergency department nurse who was required to have good communication skills and high problem-solving ability. The most important task of the coordinator was to arrange immediately a $\mathrm{CT}$ examination and to provide the cooperating nurse with information on time. Importantly, the cooperating nurse was selected from the stroke unit and was required to have a minimum of 5-year work experience. Nurses in these two positions had studied and were trained in neurological treatment theory and practice and possessed relevant nursing skills, specialized in the therapy of IVT with rtPA in emergency stroke patients.

The emergency thrombolysis cooperating nurse continually assessed and recorded the status of the patients every 15 min during the thrombolysis treatment, including 1) any bleeding at the puncture site or other vascular lesions; 2) presence of symptoms, such as disturbance of consciousness, headache, dysphasia, and hemiplegia; 3) other bleeding signs, such as gum bleeding and pharyngeal bleeding; 4) any change of vital signs, especially of blood pressure (systolic

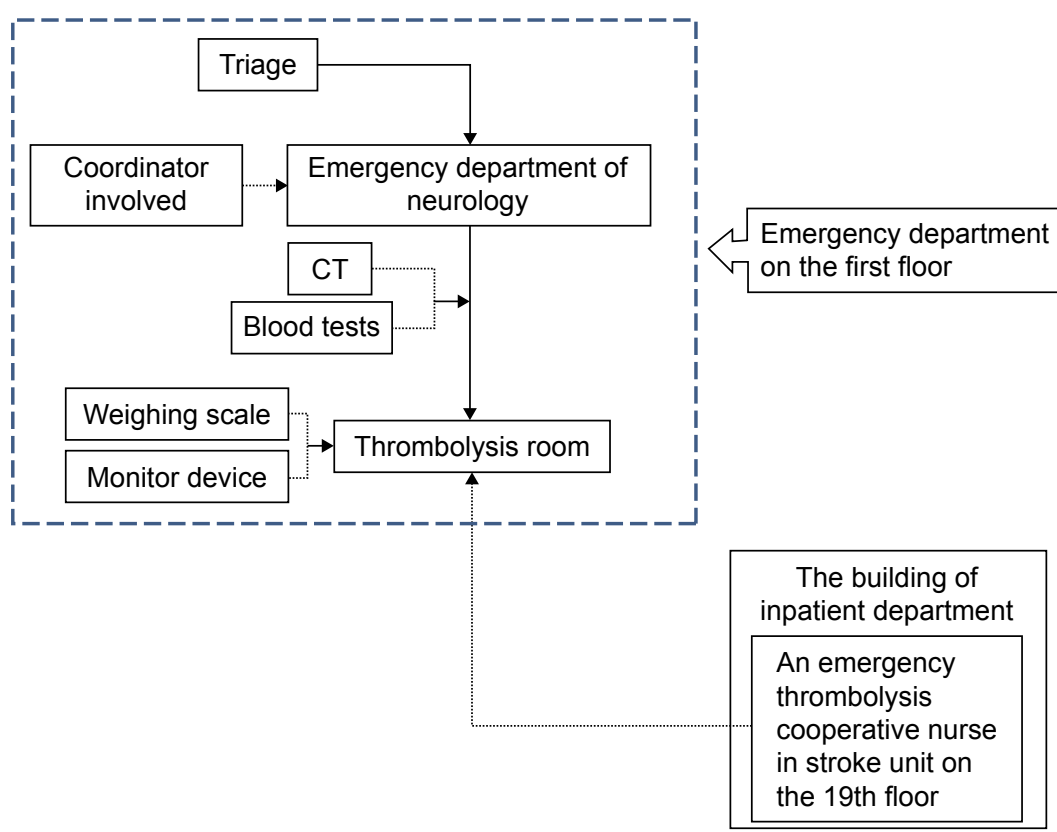

Figure I A new standardized emergency department IVT with rtPA nursing cooperation workflow from descent from the ambulance until entrance into the emergency medical unit (TI).

Abbreviations: CT, computed tomography; IVT, intravenous thrombolysis; rtPA, recombinant tissue plasminogen activators. 
pressure $\geq 200 \mathrm{mmHg}$ or diastolic pressure $\geq 110 \mathrm{mmHg}$; patient could take antihypertensive drugs by prescription but should avoid hypotension after medicine taken and maintain normal cerebral perfusion pressure by avoiding hypotension and cerebral retraction); and 5) the rate of recurrent infarction, including symptoms, such as disturbance of consciousness, dysphagia, dysphasia, and hemiplegia.

Another major element in IVT with rtPA treatment in the emergency department is the first-aid thrombolysis kit. In our study, the kit was managed by a special staff member so that the immediate application of the reagents was guaranteed. These measures are supposed to save time for the therapy once the designated nurse receives the order and arrives at the special thrombolysis unit.

The first-aid thrombolysis kit used in our investigation included 1) two bottles of rtPA (Actilyse ${ }^{\circledR}$ [Alteplase]); 2) two syringes of 60,20 , and $5 \mathrm{~mL} ; 3$ ) one indwelling needle of 22 and $24 \mathrm{G}$; 4) intravenous transfusion supplies; 5) weight conversion table; 6) thrombolysis record book; 7) the guidance for the use of the first-aid thrombolysis kit that included a staff schedule table, instructions on the procedure of handover and the administration of thrombolytic drugs, and description of the methods for weight conversion.

\section{Data collection and analyses}

All data were collected from medical records by nursing staff and included the following clinical information: the time of arrival, triage, diagnosis, onset-to-needle (OTN) time, and CT; demographics; and NIHSS scores. Three time periods, including door-to-CT initiation time, CT completion-toneedle time, and DTN time, were calculated.

Two researchers independently collected the data. Statistical analysis was conducted using the SPSS 17.0 software. The durations of the three time periods were expressed as mean \pm standard deviation. Door-to-CT initiation time, CT completion-to-needle time, and DTN time of the two groups were compared. In addition, the NIHSS scores of the two groups were compared to evaluate the availability of the new nursing procedure. In the cases the data followed a normal distribution, independent samples $t$-test was used to compare the independent means of the two groups. $\chi^{2}$ test was conducted to compare the related variables presented in percentage between the thrombolysis and nonthrombolysis groups.

\section{Results}

In total, 88 of the 689 AIS patients (402 males and 287 females, aged from 45 to 86 years) were included in the T0 group and 231 of the 712 AIS patients (406 males and
Table I General information of patients in the two groups

\begin{tabular}{llll}
\hline Groups & T0 & TI & P-value \\
\hline Age, years (mean \pm SD) & $72 \pm 3.85$ & $69 \pm 3.72$ & $>0.05$ \\
Males, n (\%) & $402(58.3)$ & $406(57.0)$ & $>0.05$ \\
\hline
\end{tabular}

Abbreviation: SD, standard deviation.

306 females, aged from 35 to 92 years) were enrolled in the T1 group (Table 1).

There was no significant difference between the two groups in the general information parameters, such as gender and age (Table 1). As listed in Table 2, the incidences receiving thrombolysis treatment in the $\mathrm{T} 1$ group were significantly higher than those in the T0 group $(P<0.01)$.

The door-to-CT initiation time, $\mathrm{CT}$ completion-to-needle time, and DTN time of the two groups were also compared. A total of 319 cases (control group: 88 cases; intervention group: 231 cases) were recruited in this study. As listed in Table 3, a significantly shorter door-to-CT initiation time, CT completion-to-needle time, and DTN time were achieved in the group treated with the newly standardized nursing cooperation workflow compared to those in the control group $(P<0.01)$.

We also compared the clinical outcomes between the two groups. As listed in Table 4, the NIHSS scores before thrombolysis in the two groups were comparable. However, after thrombolysis for $24 \mathrm{~h}$, the newly standardized nursing cooperation workflow significantly decreased the NIHSS scores $(P<0.01)$. Two weeks later, there were no significant differences in the two groups regarding NIHSS scores. In addition, the rate of lost to follow-up patients and the magnetic resonance spectrum scores $(0-1)$ during the 3 months of the follow-up period were comparable in the two groups.

\section{Discussion}

DTN time during the IVT treatment of AIS patients is critically important and determines the prognosis (recommendation grade I, evidence class A). ${ }^{15}$ In developed countries, it is possible to shorten the inhospital delay to $20 \mathrm{~min}$. Nevertheless, the DTN time could be controlled and reduced to only $<60 \mathrm{~min}$ in the Chinese health care system. ${ }^{16}$

Many health care service systems are undergoing improvement of the therapeutic process. ${ }^{17,18}$ It is also

Table 2 The rates of receiving thrombolysis treatment in the two groups

\begin{tabular}{lllll}
\hline Groups & T0 & TI & $\chi^{2}$ & P-value \\
\hline Thrombolysis, cases (\%) & $88(12.8)$ & $231(32.4)$ & 6.57 & 0.009 \\
Nonthrombolysis, cases (\%) & $601(87.2)$ & $48 I(67.6)$ & & \\
\hline
\end{tabular}


Table 3 Door-to-CT initiation, CT completion-to-needle, and DTN times in the two groups

\begin{tabular}{|c|c|c|c|c|}
\hline Time (min) & T0 $(n=88)$ & TI $(n=23 I)$ & $t$ & $P$-value \\
\hline \multicolumn{5}{|c|}{ Door-to-CT initiation time } \\
\hline Minimum & 16 & 3 & & \\
\hline Maximum & 62 & 36 & & \\
\hline Mean \pm SD & $38.67 \pm 5.21$ & $14.39 \pm 4.35$ & 21.336 & $<0.001$ \\
\hline \multicolumn{5}{|c|}{ CT completion-to-needle time } \\
\hline Minimum & 38 & 6 & & \\
\hline Maximum & 102 & 62 & & \\
\hline Mean \pm SD & $55.06 \pm 4.82$ & $30.26 \pm 3.66$ & 16.743 & $<0.001$ \\
\hline \multicolumn{5}{|l|}{ DTN time } \\
\hline Minimum & 56 & 18 & & \\
\hline Maximum & 175 & 108 & & \\
\hline Mean \pm SD & $100.43 \pm 6.05$ & $55.68 \pm 3.62$ & 18.144 & $<0.001$ \\
\hline
\end{tabular}

Note: Independent samples t-test used.

Abbreviations: CT, computed tomography; DTN, door-to-needle; SD, standard deviation.

important to establish a multilevel implementation strategy to reduce stroke thrombolysis delays in the emergency departments of hospitals. ${ }^{19,20}$ Nursing workflow was of major importance during the cooperation workflow. ${ }^{21}$ Therefore, in the present study, we explored a new standardized nursing cooperation workflow program applied in our hospital.

The new IVT nursing cooperation workflow was designed to shorten the waiting time of patients and the IVT team. It is noteworthy that the new workflow management contributed to a decrease in time for patient transfer and reduced the waiting time of patients in the emergency department before and after the IVT treatment. The key factors in this new standardized nursing cooperation workflow were as follows: 1) the coordination between the multispecialty teams and different departments was designed to reduce the delay of the treatment during the whole process; 2) emergency thrombolysis cooperating nurse. These specially trained nurses worked in shifts in the stroke unit with a 24-h on call duty to offer professional work at any time; these nurses moved from the stroke unit to the emergency department to avoid the transfer of the patients; 3 ) the thrombolysis kit, which was established for convenient use and timely therapy. In summary, this new nursing cooperation workflow improved the efficacy of thrombolysis mainly by promoting the communication among different departments.

After the application of the new standardized nursing cooperation workflow, DTN time was shortened and hospital delays were diminished. Thrombolysis treatment could be carried out as soon as possible. The implementation of the new standardized nursing cooperation workflow also increased the rate of thrombolysis therapy use. The annual work report for 2012 issued by the National Center of Quality Improvement in Stroke Care, the Ministry of Health, China, stated that only $17 \%$ of the patients in China received thrombolytic therapy within $1 \mathrm{~h}$ from the transfer from the ambulance, whereas $42 \%$ of the stroke patients received thrombolytic therapy from 1 to $2 \mathrm{~h}$ after descent from the ambulance. ${ }^{22}$ The median time from the arrival of patients to the hospital to receive thrombolytic therapy was estimated at $105 \mathrm{~min}$. The findings of this report also indicated that only $10.10 \%$ of the patients received rtPA thrombolytic therapy within $3 \mathrm{~h}$. In our study, 231 patients in the intervention group received thrombolytic therapy within $2 \mathrm{~h}$, a period which was significantly shorter than the generally required $(4.5 \mathrm{~h})$. Meanwhile, the thrombolysis rate was significantly improved (from $12.8 \%$ to $32.5 \%$ ) and a better outcome was achieved compared with that of the previous study, which accounted for $1.6 \%$ (2) and the one mentioned in the 2012 annual work report in China. ${ }^{22}$ In addition, the new standardized nursing cooperation workflow decreased the NIHSS scores of the patients receiving thrombolysis at the $24 \mathrm{~h}$ time point. Although the NIHSS scores at the 2-week point were comparable in the two groups, the newly standardized nursing cooperation workflow improved the prognosis to some extent.

There were also limitations in our study. Our results may not be completely representative of the whole country. Furthermore, the decrease in the inhospital delay might be successfully applied only in specific local conditions. Longterm effects and whether the nursing cooperation workflow is suitable for other undeveloped countries are still not validated. This study was a retrospective, nonrandomized, "open" type of analysis. Furthermore, because the treatments in the

Table 4 Comparison of clinical outcomes in the two groups

\begin{tabular}{|c|c|c|c|c|}
\hline Outcome indicators & T0 $(n=88)$ & TI $(n=23 I)$ & $t / \chi^{2}$ & P-value \\
\hline NIHSS scores before thrombolysis (mean $\pm \mathrm{SD}$ ) & $6.97 \pm 3.98$ & $7.00 \pm 3.89$ & -0.078 & 0.938 \\
\hline NIHSS scores $24 \mathrm{~h}$ after thrombolysis, (mean $\pm \mathrm{SD}$ ) & $3.33 \pm 2.09$ & $2.60 \pm 1.66$ & 2.952 & 0.004 \\
\hline NIHSS scores 2 weeks after thrombolysis (mean \pm SD) & $2.25 \pm 1.01$ & $2.21 \pm 1.02$ & 0.331 & $0.74 I$ \\
\hline Lost to follow-up during the 3 months, cases (\%) & $3(3.4)$ & $8(3.5)$ & 0.001 & 0.981 \\
\hline mRs scores $(0-1)$ during the 3 months, cases (\%) & $85(96.5)$ & $223(96.8)$ & 0.030 & 0.863 \\
\hline
\end{tabular}

Note: Independent samples $t$-test used.

Abbreviations: NIHSS, National Institutes of Health Stroke Scale; SD, standard deviation; mRs, magnetic resonance spectrum. 
two groups were conducted in different, nonoverlapping time frames (2014 vs 2015), other temporal-related differences apart from physician performance could have had an influence.

\section{Conclusion}

The new standardized nursing cooperation workflow significantly reduced the stroke thrombolysis delays in our emergency department. Therefore, we recommend that health care staff in other hospitals receive educational in-service training, which would be beneficial for the wide application of this new approach.

\section{Disclosure}

The authors report no conflicts of interest in this work.

\section{References}

1. Yang W, Li JP, Zhang Y, et al. Association between body mass index and all-cause mortality in hypertensive adults: results from the China Stroke Primary Prevention Trial (CSPPT). Nutrients. 2016;8(6):E384.

2. Wang $\mathrm{Y}$, Liao X, Zhao X, et al. Using recombinant tissue plasminogen activator to treat acute ischemic stroke in China: analysis of the results from the Chinese National Stroke Registry (CNSR). Stroke. 2011;42(6): $1658-1664$

3. Feigin VL, Forouzanfar MH, Krishnamurthi R, et al. Global and regional burden of stroke during 1990-2010: findings from the Global Burden of Disease Study 2010. Lancet. 2014;383(9913):245-254.

4. Yang G, Wang Y,Zeng Y, etal. Rapid health transition in China, 1990-2010: findings from the Global Burden of Disease Study 2010. Lancet. 2013; 381(9882):1987-2015.

5. Fonarow GC, Smith EE, Saver JL, et al. Timeliness of tissue-type plasminogen activator therapy in acute ischemic stroke: patient characteristics, hospital factors, and outcomes associated with door-to-needle times within 60 minutes. Circulation. 2011;123(7):750-758.

6. Powers WJ, Derdeyn CP, Biller J, et al. 2015 American Heart Association/ American Stroke Association focused update of the 2013 guidelines for the early management of patients with acute ischemic stroke regarding endovascular treatment: a guideline for healthcare professionals from the American Heart Association/American Stroke Association. Stroke. 2015; 46(10):3020-3035.

7. Fonarow GC, Zhao X, Smith EE, et al. Door-to-needle times for tissue plasminogen activator administration and clinical outcomes in acute ischemic stroke before and after a quality improvement initiative. JAMA. 2014;311(16):1632-1640.
8. Saver JL, Fonarow GC, Smith EE, et al. Time to treatment with intravenous tissue plasminogen activator and outcome from acute ischemic stroke. JAMA. 2013;309(23):2480-2488.

9. Casolla B, Bodenant M, Girot M, et al. Intra-hospital delays in stroke patients treated with rt-PA: impact of preadmission notification. J Neurol. 2013;260(2):635-639.

10. Huang Q, Ma QF, Feng J, et al. Factors associated with in-hospital delay in intravenous thrombolysis for acute ischemic stroke: lessons from China. PLoS One. 2015;10(11):e0143145.

11. Reynolds SS, Murray LL, McLennon SM, Bakas T. Implementation of a stroke competency program to improve nurses' knowledge of and adherence to stroke guidelines. J Neurosci Nurs. 2016;48(6):E1.

12. Ren X, Zhang X, Du Y, Li X, Liu S. [Research on the nursing stroke patients during past 10 years]. Zhonghua Hu Li Za Zhi. 1991(8):35-37. Chinese.

13. Lee RL, Mok ES. Seeking harmony in the provision of care to the stroke-impaired: views of Chinese family caregivers. J Clin Nurs. 2011; 20(9-10): 1436-1444.

14. Quaglini S, Cavallini A, Gerzeli S, Micieli G, Group GS. Economic benefit from clinical practice guideline compliance in stroke patient management. Health Policy. 2004;69(3):305-315.

15. Thortveit ET, Boe MG, Ljostad U, Mygland A, Tveiten A. Organizational changes aiming to reduce iv tPA door-to-needle time. Acta Neurol Scand. 2014;130(4):248-252.

16. Meretoja A, Strbian D, Mustanoja S, Tatlisumak T, Lindsberg PJ, Kaste M. Reducing in-hospital delay to 20 minutes in stroke thrombolysis. Neurology. 2012;79(4):306-313.

17. Ning W, Yan J. The application of medical failure mode and effective analysis of thrombolysis in stroke. J Nurs. 2015;5:63-65.

18. Zhu G, Liu Y, Wang Y, Bi X, Baudry M. Different patterns of electrical activity lead to long-term potentiation by activating different intracellular pathways. $J$ Neurosci. 2015;35(2):621-633.

19. Ford AL, Williams JA, Spencer M, et al. Reducing door-to-needle times using Toyota's lean manufacturing principles and value stream analysis. Stroke. 2012;43(12):3395-3398.

20. Meretoja A, Weir L, Ugalde M, et al. Helsinki model cut stroke thrombolysis delays to 25 minutes in Melbourne in only 4 months. Neurology. 2013;81(12):1071-1076.

21. Studnek JR, Bentley M, Crawford JM, Fernandez AR. An assessment of key health indicators among emergency medical services professionals. Prehosp Emerg Care. 2010;14(1):14-20.

22. State of the Ministry of Health Stroke Chinese Medicine Quality Control Center. 2012 Annual Work Summary. Vol. 67. 2012:73.
Neuropsychiatric Disease and Treatment

\section{Publish your work in this journal}

Neuropsychiatric Disease and Treatment is an international, peerreviewed journal of clinical therapeutics and pharmacology focusing on concise rapid reporting of clinical or pre-clinical studies on a range of neuropsychiatric and neurological disorders. This journal is indexed on PubMed Central, the 'PsycINFO' database and CAS,

\section{Dovepress}

and is the official journal of The International Neuropsychiatric Association (INA). The manuscript management system is completely online and includes a very quick and fair peer-review system, which is all easy to use. Visit http://www.dovepress.com/testimonials.php to read real quotes from published authors. 